\title{
Avaliação de fungicidas, óleos essenciais e agentes biológicos no controle de Amphobotrys ricini em mamoneira (Ricinus communis L.)
}

\author{
Haroldo Antunes Chagas ${ }^{1 *}$, Marco Antonio Basseto ${ }^{2}$, Daniel Dias Rosa ${ }^{2}$, Eder Victor Braganti Toppa ${ }^{1}$, Edson Luiz \\ Furtado $^{2 * *}$, Mauricio Dutra Zanotto ${ }^{1}$
}

\begin{abstract}
${ }^{1}$ Departamento de Produção Vegetal Setor Agricultura - Faculdade de Ciências Agronômicas, UNESP. CP 237, Botucatu, SP, $18610-307$. ${ }^{2}$ Departamento de Produção Vegetal Setor Defesa Fitossanitária - Faculdade de Ciências Agronômicas, UNESP. CP 237, Botucatu, SP, $18610-307$. *Bolsista CAPES, ${ }^{* *}$ Bolsista CNPq Autor para correspondência: Haroldo Antunes Chagas (haroldo.antunes@yahoo.com.br) Data de chegada: 10/10/2013. Aceito para publicação em: 03/02/2014.
\end{abstract}

\section{RESUMO}

Chagas, H.A.; Basseto, M.A.; Rosa, D.D.; Toppa, E.V.B.; Furtado, E.L.; Zanotto, M.D.. Avaliação de fungicidas, óleos essenciais e agentes biológicos no controle de Amphobotrys ricini em mamoneira (Ricinus communis L.). Summa Phytopathologica, v.40, n.1, p.42-48, 2014.

A mamoneira (Ricinus communis L.) é uma espécie oleaginosa tropical, sendo o óleo extraído de suas sementes um dos mais versáteis da natureza e com inúmeras aplicações industriais. Embora ainda seja uma espécie rústica, ela está sujeita a diversas doenças, dentre elas o mofo-cinzento, causada pelo fungo Amphobotrys ricini. O melhoramento genético seria a melhor alternativa para o controle da doença, mas demanda tempo para se obter cultivares resistentes. Dessa maneira, o uso de métodos de controle baseado em métodos químicos, alternativos ou biológicos mostra-se viável no curto prazo. O objetivo do trabalho foi estudar a eficiência do controle do mofo-cinzento, na cultura da mamoneira, utilizando-se de métodos químico, alternativo e biológico. Assim, procurou-se avaliar, tanto in vitro, quanto in vivo a eficiência de controle do patógenos utilizando-se de fungicidas, óleos essenciais e agentes de controle biológico. Quanto a inibição do crescimento micelial do patógeno in vitro os melhores tratamentos com os óleos essenciais foram os com a base de Cymbopogon martini e Cynnamomum zeylanicum, nas cinco concentrações testadas. Em ambos os óleos, o diâmetro médio das colônias ficou em $0,7 \mathrm{~cm}$ contra a média de 4,79 $\mathrm{cm}$ da testemunha. Com os fungicidas, nas quatro concentrações testadas, os mais eficientes foram os ingredientes ativos tiofanato metílico, carbendazim, tebuconazole e iprodione. $\mathrm{O} \mathrm{ED}_{50}$ destes fungicidas ficou $<1 \mathrm{uL} / \mathrm{L}$, atestando $100 \%$ de inibição do crescimento micelial em todas as concentrações. Quanto à inibição da germinação dos conídios de A. ricini, os fungicidas tebuconazole e clorotalonil foram os melhores em todas as concentrações testadas, sendo a média dos conídios germinados destes fungicidas de 0,0 e $0,15 \%$, respectivamente, contra $100 \%$ da testemunha. No campo, o tratamento com o fungicida iprodione foi o melhor quanto ao controle da doença quando comparados com os tratamentos biológico e alternativo. Em condições de campo, a severidade média da doença com o tratamento com iprodione foi de $15,76 \%$ contra $95,81 \%$ na testemunha inoculada.

Palavras-chave adicionais: mamona, controle, doença, extratos vegetais e mofo cinzento.

\section{ABSTRACT}

Chagas, H.A.; Basseto, M.A.; Rosa, D.D.; Toppa, E.V.B.; Furtado, E.L.; Zanotto, M.D..Evaluation of fungicides, essential oils and biological agents on Amphobotrys ricini control in castor bean (Ricinus communis L.). Summa Phytopathologica, v.40, n.1, p.42-48, 2014.

The castor bean (Ricinus communis L.) is a tropical oilseed species, and the oil extracted from its seeds is one of the most versatile oils in the nature, showing various industrial uses. Even though it is a rustic species, the castor bean is subjected to several diseases such as the gray mold, caused by the fungus Amphobotrys ricini. Genetic breeding would be the best alternative for the disease control, but a long time is required to obtain resistant cultivars. Thus, the use of control strategies based on chemical, alternative or biological methods shows viable in the short term. The aim of this study was to investigate gray mold control efficiency, in castor bean crop, using chemical, alternative and biological methods. The pathogen control efficiency was evaluated both in vitro and in vivo using fungicides, essential oils and biological control agents. As regards the in vitro inhibition of the pathogen mycelial growth, the best treatments with essential oils were those based on C. martini and
C. zeylanicum at all five tested concentrations. For both oils, the average diameter of colonies was $0.7 \mathrm{~cm}$ against $4.79 \mathrm{~cm}$ for the control treatment. For the fungicides, at all four tested levels, the most efficient active ingredients were methyl tiophanate, carbendazim, tebuconazole and iprodione. The $\mathrm{ED}_{50}$ of these fungicides was $<1 \mathrm{uL} / \mathrm{L}$, yielding $100 \%$ mycelial growth inhibition at all concentrations. As to the inhibition of $A$. ricini conidium germination, the fungicides tebuconazole and chlorotanolyl were the best at all tested concentrations, and the average of germinated conidia with these fungicides was 0.0 and $0.15 \%$, respectively, against $100 \%$ for the control treatment. In the field, treatment with the fungicide iprodione was the best for the disease control when compared to biological and alternative treatments. Under field conditions, the average disease severity for the treatment with iprodione was $15.76 \%$ against $95.81 \%$ for the inoculated control.

Additional keywords: castor bean, control, disease, vegetal extracts and gray mold. 
A mamoneira (Ricinus communis L.), também conhecida como carrapateira ou rícino, é uma espécie de origem tropical que vegeta naturalmente desde a longitude $40^{\circ}$ Norte até $40^{\circ} \mathrm{Sul}$. O óleo extraído de suas sementes é um dos mais versáteis da natureza, com inúmeras aplicações industriais (6).

Embora seja uma planta com grande capacidade de adaptação às mais diferentes regiões do mundo, a mamoneira está sujeita a doenças causadas por diversos microorganismos, os quais causam grandes prejuízos econômicos, principalmente quando as condições climáticas lhes são favoráveis $(9,22)$.

Entre essas doenças, pode-se destacar o mofo cinzento, causado pelo fungo Amphobotrys ricini (Buchw.) Hennebert, como uma das mais importantes, pois causa grandes prejuízos à produção, destruindo inflorescências e racemos, e assim reduzindo a produção de óleo pela diminuição dos frutos colhidos (12).

O melhoramento genético vegetal é um mecanismo que visa a obtenção de materiais que apresentem características agronômicas superiores as de seus genitores, sendo que dentro dessas características destacam-se aumento de produtividade, precocidade de desenvolvimento e resistência ao ataque de doenças, entre outras, sendo tudo isso possível através da seleção de genótipos superiores. Entretanto, apesar do melhoramento mostrar-se como alternativa ideal no controle do mofo cinzento, o mesmo esbarra em fatores primordiais para a cultura, como fonte de resistência para a doença e, também, em tempo hábil para os cruzamentos e testes de progênies. Dessa maneira, tornase interessante um direcionamento das pesquisas para o controle alternativo e químico dessa doença a curto prazo.

O controle químico tem sido empregado na agricultura há cerca de 200 anos para proteger as plantas de doenças provocadas, principalmente, por ataques de fungos. Do início pequeno e primitivo, voltado principalmente para proteção de sementes de cereais e videiras, o número de culturas e das doenças tratadas, a variedade de compostos químicos disponíveis, a área e frequência de seu uso e a eficiência dos tratamentos aumentaram extraordinariamente, principalmente depois da segunda guerra mundial. Foi só na última década que a quantidade e a variedade de tratamentos com fungicidas, no mundo inteiro, atingiu algum grau de estabilidade e maturidade (3).

Dentre os agentes de controle biológico, o fungo filamentoso Clonostachys rosea surgiu como um eficaz e versátil antagonista em estudos no final da década de 80, para controlar Botrytis cinerea (28).

Outro agente de controle biológico utilizado são os fungos do gênero Trichoderma que apresentam relatos de controle em parte aérea de plantas tais como: Venturia spp., Botrytis spp., Crinipellis perniciosa, entre outros; sendo que o seu potencial foi descoberto na década de 30 (30).

Outro método de controle bastante interessante seria o uso de compostos secundários presentes no extrato bruto ou em óleo essencial de plantas medicinais, podendo constituir em mais uma potencial forma de controle alternativo de doenças em plantas (23).

Dessa maneira, esses tipos de controle apresentam-se como uma alternativa, principalmente no que diz respeito a cultura da mamoneira, que desempenha papel importante como fonte de renda para a agricultura familiar,destacadamente na região nordeste.

O objetivo do trabalho foi estudar métodos de controle ao fungo $A$. ricini, agente causal do mofo cinzento na mamoneira, tanto em condições de ensaio in vitro, quanto in vivo.

\section{MATERIAL E MÉTODOS}

\section{Avaliação in vitro do crescimento micelial do isolado de Amphobotrys ricini, utilizando diferentes concentrações de óleos essenciais e fungicidas}

O experimento foi realizado no Laboratório de Patologia Florestal do Setor de Defesa Fitossanitária do Departamento de Produção Vegetal. Foram utilizados sete óleos essenciais das seguintes espécies: Eucaliptus citriodora (eucalipto), Cymbopogon martini (palma-rosa), Cinnamomum zeylanicum (canela), Mentha piperita (menta), Citrus limom (limão), Melaleuca alternifolia (tea tree), Azadirachta indica (nim) e testemunha. Foram preparadas 200 placas de Petri contendo meio BDA com 5 diferentes concentrações dos óleos, a saber: 1000; 2000; 3000; 4000 e 5000) $\mu \mathrm{L} / \mathrm{L}$. Repicaram-se para o meio BDA, contendo os tratamentos, discos de $0,7 \mathrm{~cm}$ de diâmetro do isolado do fungo (L3) cedido pela Embrapa Algodão de Campina Grande na Paraíba, da borda das colônias com 11 dias de idade e transferidos para o centro das placas e mantidas em câmara do tipo BOD, à $25^{\circ} \mathrm{C}$, com fotoperíodo de $12 \mathrm{~h}$, até o $6^{\circ}$ dia.

A avaliação foi realizada por meio da medição diária dos diâmetros (cm) das colônias em dois sentidos perpendiculares entre si, tomando-se como valor de crescimento a média das duas medidas. O delineamento experimental utilizado foi inteiramente casualizado, em esquema fatorial $8 \times 5$. Os resultados foram submetidos ao teste de média de Tukey, a 5\% de probabilidade.

Foram comparados nove fungicidas comerciais (Tabela 1) de diferentes grupos químicos, tanto usados para tratamento de sementes quanto para parte aérea, para controle do fungo Botrytis cinerea.

Para produção do inóculo, discos de micélio do isolado (L3), de $0,7 \mathrm{~cm}$ de diâmetro, foram transferidos para o centro de placas de Petri contendo meio BDA e, posteriormente, incubados sob condições controladas (temperatura de $25^{\circ} \mathrm{C}$ e fotoperíodo de 12 horas), até a colonização do fungo atingir o diâmetro total da placa.

Para o preparo do meio de cultura com fungicida, cada produto foi adicionado ao meio de cultura BDA. Cada produto utilizado foi diluído em água destilada autoclavada, sob fluxo laminar. Primeiro foi feita uma suspensão estoque I para cada fungicida, ou seja $1 \mathrm{~g}$ de i.a. de cada produto comercial, que podia variar de acordo com a porcentagem para cada produto em $100 \mathrm{~mL}$ de água, obtendo-se a concentração de 10.000 ppm. A partir desta solução estoque, foram feitas diluições em série, transferindo-se $10 \mathrm{~mL}$ da solução estoque para $90 \mathrm{~mL}$ de água, obtendo-se a solução estoque II (1.000 ppm), e transferindo-se $10 \mathrm{~mL}$ da solução II para $90 \mathrm{~mL}$ de água, obtendo-se a solução estoque III (100 ppm) e a última diluição de $10 \mathrm{~mL}$ da solução III para $90 \mathrm{~mL}$ de água, obtendo-se assim a solução estoque IV (10 ppm). De cada uma dessa soluções estoques, foram retirados $10 \mathrm{~mL}$ e transferidos para 90 $\mathrm{mL}$ de meio BDA fundente $\left(45-47^{\circ} \mathrm{C}\right)$, obtendo-se assim os meios nas diluições de 1000, 100, 10 e 1 ppm, respectivamente.

Discos do meio BDA, de $0,7 \mathrm{~cm}$ de diâmetro, contendo micélio do fungo foram transferidos para o centro das placas de Petri com meio BDA com os fungicidas. As testemunhas consistiram de discos de micélio colocados em meio BDA sem fungicida. A incubação ocorreu sob condições controladas, temperatura de $25^{\circ} \mathrm{C}$ e fotoperíodo de 12 horas.

Cada tratamento foi representado por cinco placas de Petri com 4 concentrações de cada um dos fungicida, mais a testemunha. $\mathrm{O}$ delineamento foi inteiramente casualizado, esquema fatorial $10 \mathrm{X} 4$, ou seja, 10 tratamentos, quatro concentrações (1, 10, 100 e 1000 ppm) e cinco repetições, sendo cada repetição uma placa de Petri.

A avaliação foi realizada diariamente e terminou quando a 
Tabela 1. Fungicidas testados quanto à eficiência in vitro no controle de Amphobotrys ricini Botucatu-SP.

\begin{tabular}{|c|c|c|c|c|}
\hline Ingrediente ativo (i.a) & Grupo químico & Classe & Formulação & Concentração \\
\hline Azoxystrobina & Estrobilurina & sistêmico & WG & $500 \mathrm{~g} / \mathrm{Kg}$ \\
\hline Clorotalonil & Isoftalonitrila & contato & PM & $750 \mathrm{~g} / \mathrm{Kg}$ \\
\hline Tiofanato metílico & Benzamidozóis & sistêmico & PM & $700 \mathrm{~g} / \mathrm{Kg}$ \\
\hline Carbendazim & Benzamidazóis & sistêmico & $\mathrm{SC}$ & $500 \mathrm{~g} / \mathrm{L}$ \\
\hline Tebuconazole & Triazóis & sistêmico & $\mathrm{CE}$ & $200 \mathrm{~g} / \mathrm{L}$ \\
\hline Mancozeb & Ditiocarbamatos & contato & PM & $800 \mathrm{~g} / \mathrm{Kg}$ \\
\hline Ipridione & Dicarboximida & contato & $\mathrm{SC}$ & $500 \mathrm{~g} / \mathrm{L}$ \\
\hline Procimidone & Dicarboximida & sistêmico & PM & $500 \mathrm{~g} / \mathrm{Kg}$ \\
\hline Carboxin + thiram & Carboxanilida + dimetilditiocarbamato & sistêmico + contato & $\mathrm{SC}$ & $200+200 \mathrm{~g} / \mathrm{L}$ \\
\hline
\end{tabular}

SC- suspensão concentrada; PM- pó molhavel; CE- concentração emulsionável; WG- granulado

colonização do meio testemunha atingiu próximo ao diâmetro total das placas $(8,5 \mathrm{~cm})$, o que levou por volta de 11 dias. Com o auxílio de uma régua, mediram-se, em dois sentidos perpendiculares, os diâmetros da colônia de cada placa de Petri, com os respectivos produtos e concentrações, comparando-os ao crescimento médio das testemunhas. Assim, calculou-se o valor da porcentagem de inibição do crescimento micelial (PIC) (15).

\section{$\mathrm{PIC}=\underline{\text { Crescimento da testemunha }- \text { Crescimento tratamento }} \times 100$ Crescimento da testemunha}

Para a análise estatística, as médias dos dados foram comparadas pelo teste de Tukey a 5\% de probabilidade.

Correlacionando a porcentagem de inibição com o logarítmo da concentração do fungicida, foi obtido, graficamente, o valor aproximado da dose efetiva mediana $\left(\mathrm{ED}_{50}\right)$ ou seja, a concentração do produto químico necessária pra inibir em $50 \%$ o crescimento micelial do fungo (7).

Após o cálculo da $\mathrm{ED}_{50}$, os fungicidas foram classificados em quatro categorias de eficiência, segundo escala de Bollen \& Fucks (2), Edgington et al. (7) e Kataria \& Grover (10), em que:

a) $\operatorname{ED} 50<1 \mu \mathrm{L} / \mathrm{L}$ : altamente eficiente $(\mathrm{AE})$;

b) ED50 entre 1-10 $\mu \mathrm{L} / \mathrm{L}$ : moderadamente eficiente (ME);

c) ED50 entre 10-50 $\mu \mathrm{L} / \mathrm{L}$ : pouco eficiente (PE);

d) ED50>50 $\mu \mathrm{L} / \mathrm{L}$ : ineficiente (I).

Efeito de diferentes tratamentos na severidade de Amphobotrys ricini versus mamoneira, em ensaio conduzido no campo

Com base nos resultados obtidos nas avaliações in vitro, os dois melhores tratamentos com óleos essenciais foram utilizados no campo. Para os fungicidas, apesar dos resultados diferirem quanto aos melhores produtos devido ao fato de ter ocorrido resultados diferentes para as avaliações de crescimento micelial e germinação de esporos, optou-se por utilizar os fungicidas iprodione e procimidone, por estes já serem empregados na cultura. Não houve diferenças significativas entre a cultivar AL Guarany e o híbrido Lyra, dessa maneira os materiais foram avaliados juntos.

O experimento foi conduzido na área pertencente ao Departamento de Recursos Naturais do Setor de Ciências Ambientais, da Fazenda Experimental Lageado, no município de Botucatu - SP. O delineamento experimental utilizado consistiu em blocos inteiramente casualizados num fatorial 6 X 2, correspondendo a seis tratamentos e dois genótipos + testemunhas, sendo quatro blocos compostos por quatro plantas representando uma parcela de $2 \mathrm{~m}^{2}$. A área apresentava dimensão de $24 \mathrm{~m}$ de comprimento e $8 \mathrm{~m}$ de largura, o espaçamento utilizado foi de $0,5 \mathrm{~m}$ entre plantas e $1 \mathrm{~m}$ entre linhas.

O material utilizado foi a cultivar AL-Guarany, e o Híbrido Lyra utilizados nos programas de melhoramento de mamona da Faculdade de Ciências Agrárias - UNESP, Campus de Botucatu - SP.

Os tratos culturais, adubação de plantio, cobertura, controle de plantas daninhas e pragas foram realizados nas épocas propícias. Os tratamentos adotados foram:

1) Suspensão de Clonostachys rosea na concentração de $1 \times 10^{6}$ conídios. $\mathrm{mL}^{-1}$;

2) Suspensão de Trichoderma harzianum na forma do produto comercial Trichodermil, produzido pela empresa Itaforte S/A, utilizando-se a dosagem de 2 L.ha' ${ }^{-1}$;

3) Óleo de canela (Cinnamomum zeylanicum) na dosagem de $1000 \mathrm{uL} / \mathrm{L}$ de água;

4) Óleo de palma-rosa (Cympobogon martini) na dosagem de $1000 \mathrm{uL} / \mathrm{L}$ de água;

5) Fungicida procimidone, na dosagem de $100 \mathrm{~g}$ de i.a ou 200 $\mathrm{mL}$ de p.c./ $100 \mathrm{~L}$ de água;

6) Fungicida iprodione, na dosagem de $100 \mathrm{~g}$ de i.a ou $200 \mathrm{~mL}$ de p.c./100 L de água;

7) Testemunha inoculada;

8) Testemunha não inoculada.

Foram realizadas duas aplicações de cada um dos produtos dos tratamentos com intervalo de 1 semana entre as aplicações, sendo elas no início da formação dos frutos, com pulverizadores manuais. Uma semana após a segunda aplicação dos tratamentos, foi realizada uma inoculação de uma suspensão de esporos de $1 \times 10^{6}$ conídios/mL do isolado (L3) de Amphobotrys ricini, utilizandose um pulverizador manual. Os racemos dos tratamentos e da testemunha inoculada foram envolvidos por sacos plásticos com algodão umedecidos e sacos de papel, durante 3 dias, agindo como uma câmara úmida, para favorecer o desenvolvimento do fungo. A testemunha não inoculada permaneceu envolvida por sacos de papel durante todo o experimento, evitando assim a possibilidade de receber inóculo.

A avaliação da severidade da doença do experimento foi realizada com a escala diagramática desenvolvida por Chagas et al. (5) durante 3 semanas. Os dados coletados foram submetidos a análise estatística e as médias dos dados comparadas entre si por meio do teste de Tukey a $5 \%$. 


\section{RESULTADO E DISCUSSÕES}

Avaliação in vitro do crescimento micelial do isolado de Amphobotrys ricini, utilizando diferentes concentrações óleos essenciais e fungicidas

Em certas concentrações, os óleos essenciais influenciaram o crescimento de $A$. ricini.

$\mathrm{Na}$ concentração de $1000 \mu \mathrm{L} / \mathrm{L}$, os óleos das espécies $C$. limon $(6,72 \mathrm{~cm}), M$. piperita $(4,43 \mathrm{~cm})$ e $M$. alternifolia $(4,40 \mathrm{~cm})$ foram os que menos inibiram o crescimento do fungo, quando comparados com E. citriodora $(2,01 \mathrm{~cm}), A$. indica $(2,5 \mathrm{~cm})$, Cinnamomum zeylanicum $(0,7 \mathrm{~cm})$ e $C$. martini $(0,7 \mathrm{~cm})$.

Quando comparados na concentração de $2000 \mu \mathrm{L} / \mathrm{L}$, os óleos de $C$. martini e C. zeylanicum mantiveram o efeito inibitório no crescimento micelial do fungo, apresentando para ambos valores de $0,7 \mathrm{~cm}$. Os óleos de E. citriodora e M. piperita apresentaram uma redução no crescimento micelial de $0,92 \mathrm{~cm}$ e $3,66 \mathrm{~cm}$ respectivamente.

Aos $3000 \mu \mathrm{L} / \mathrm{L}$, os óleos de M. alternifolia, C. martini e $C$. zeylanicum inibiram totalmente o crescimento micelial do fungo. Foram seguidos por M. piperita $(0,76 \mathrm{~cm})$ e E. citriodora $(0,80 \mathrm{~cm})$.

$\mathrm{Na}$ concentração de $4000 \mu \mathrm{L} / \mathrm{L}$, verficou-se que o crescimento micelial foi de $1,99 \mathrm{~cm}$ para o óleo de $M$. alternifólia. Já o óleo de $C$. limon não apresentou inibição do fungo $(5,77 \mathrm{~cm})$ e os outros óleos apresentaram valores próximos. Na concentração de $5000 \mu \mathrm{L} / \mathrm{L}$, o comportamento foi semelhante ao da concentração de $4000 \mu \mathrm{L} / \mathrm{L}$.

Esses resultados estão em conformidade com o trabalho de Souza et al. (27) que verificaram que $S$. aromaticum e C. zeylanicum foram eficientes na inibição micelial dos fungos Rhizopus sp., Penicillium sp., Eurotpium repens e Aspergillus niger, nas concentrações de 200 e 800 $\mu \mathrm{g} / \mathrm{mL}$, e que o segundo óleo inibiu totalmente os fungos na primeira concentração. Outros trabalhos com óleo essencial de C. zeylanicum mostraram a eficiência em inibir outros fungos. Singh et al. (25) e Montes-Belmont \& Carvajal (17) verificam inibição de A. niger, A. fumigatus, $A$. nidulans e $A$. flavus. Resultados similares da acão inibitória de C. zeyalnicum no crescimento micelial de Alternaira solani foram observados no trabalho de Abreu (1) em todas as concentrações utilizadas $250,500,750$ e $1000 \mu \mathrm{l} / \mathrm{L}$, bem como o efeito inferior do óleo essencial de limão (Citrus limon).

Quanto ao óleo de C. martini, os resultados encontrados neste trabalho também condizem com os encontrados por Abreu (1) e Singh et al. (24) quando constataram que esse óleo na concetração de $1 \%$ foi eficiente contra Helminthosporium oryzae, do que fungicidas sintéticos. Neste trabalho verificou-se que E. citriodora também apresentou bom resultado, confirmando obtidos por Salgado et al. (21), os quais trabalharam com óleos essenciais de diferentes espécies de Eucalyptus e que apresentaram efeito fungistático na concentração de $500 \mathrm{mg} / \mathrm{Kg}$, in vitro, no controle de Fusarium oxysporum Schelecht., Bipolaris sorokiniana Shoemaker e Botryitis cinerea Pers.

O que pode ser observado no presente trabalho, é que em todas as concentrações os óleos de C. zeylanicum e C. martini não permitiram o crescimento do fungo (Tabela 2). O óleo de $C$. limon não inibiu o crescimento do fungo, apresentando efeito contrário e favorecendo o crescimento quando comparado com a testemunha, crescendo em toda a área da placa no sexto dia de avaliação. Isto deve ter ocorrido, possivelmente devido a algum constituinte do óleo que possa ter favorecido esse rápido desenvolvimento. Em relação ao óleo de $C$. limon, este resultado está de acordo com o observado por Norman et al. (18) e McCalley \& Torres-Grifol (15), que relataram a pouca eficiência na inibição de microorganismos, ou uma possível especificidade por algum fungo ou bactéria.

As porcentagens de inibição (PICs) dos nove fungicidas testados in vitro, a 1, 10100 e $1000 \mu \mathrm{L} / \mathrm{L}$, para o isolado de $A$. ricini, podem ser visualizadas na Tabela 3. Também foram obtidos através dos PICs, a diferentes concentrações, os respectivos $\mathrm{ED}_{50}$ dos respectivos fungicidas (Tabela 4).

Para os fungicidas tebuconazole, carbendazim, tiofanato metílico e iprodione não foi possível estabelecer o $\mathrm{ED}_{50}$, devido ao fato de que em todas as concentrações utilizadas houve inibição de $100 \%$ do crescimento micelial do fungo. Apenas pode-se afirmar que o $\mathrm{ED}_{50}$ é menor que $1 \mu \mathrm{L} / \mathrm{L}$.

Os fungicidas azoxystrobina, mancozeb e carboxim + thiran foram considerados ineficientes $\left(\mathrm{ED}_{50}>50 \mu \mathrm{L} / \mathrm{L}\right)$, diferente do que foi observado por Fischer (8), que trabalhou com o fungo Nectria haematococca. Já os fungicidas tiofanato metílico, carbendazim, tebuconazole, iprodione e procimidione foram altamente eficientes $\left(\mathrm{ED}_{50}<1 \mu \mathrm{L} / \mathrm{L}\right)$.

Segundo Kimura (11), em seu trabalho ocorreu a insensibilidade do isolado de Botrytis cinerea ao fungicida tiofanato metílico, que confirma a ocorrência de resistência cruzada para esse grupo de fungicidas, com abundante desenvolvimento micelial na concentração de $1000 \mu \mathrm{L} / \mathrm{L}$ do fungicida no meio BDA, demonstrando alto nível de resistência. Porém, neste trabalho ocorreu o inverso, não houve crescimento

Tabela 2. Desenvolvimento de Amphobotrys ricini sob ação de diferentes óleos essenciais. Botucatu-SP, 2009.

\begin{tabular}{|c|c|c|c|c|c|}
\hline \multirow{3}{*}{ Tratamentos } & \multicolumn{5}{|c|}{ Concentrações $(\mu \mathrm{L} / \mathrm{L})$} \\
\hline & 1000 & 2000 & 3000 & 4000 & 5000 \\
\hline & \multicolumn{5}{|c|}{ Diâmetro $(\mathrm{cm})$} \\
\hline Testemunha & $4,75 \mathrm{Ba}$ & $4,72 \mathrm{ABa}$ & $4,92 \mathrm{Aa}$ & $4,91 \mathrm{Aa}$ & $4,64 \mathrm{Aa}$ \\
\hline Cymbopogon martini & $0,7 \mathrm{Da}$ & $0,7 \mathrm{Ea}$ & $0,7 \mathrm{Ca}$ & $0,7 \mathrm{Ba}$ & $0,7 \mathrm{Ba}$ \\
\hline Melaleuca alternifolia & $4,4 \mathrm{Ba}$ & $3,66 \mathrm{BCa}$ & $0,7 \mathrm{Cc}$ & $1,99 \mathrm{Bb}$ & $1,03 \mathrm{Bc}$ \\
\hline Citrus limon & $6,72 \mathrm{Aa}$ & $6,06 \mathrm{Aa}$ & $5,9 \mathrm{Aa}$ & $5,77 \mathrm{Aa}$ & $6,05 \mathrm{Aa}$ \\
\hline Mentha piperita & $4,43 \mathrm{Ba}$ & $2,44 \mathrm{Db}$ & $0,76 \mathrm{Cc}$ & $0,74 \mathrm{Bc}$ & $0,76 \mathrm{Bc}$ \\
\hline Azadirachta indica & $2,5 \mathrm{Cab}$ & $2,65 \mathrm{CDa}$ & $2,06 \mathrm{Bab}$ & $1,7 \mathrm{Bbc}$ & $1,1 \mathrm{Bc}$ \\
\hline Cynnamomum zeylanicum & $0,7 \mathrm{Da}$ & $0,7 \mathrm{Ea}$ & $0,7 \mathrm{Ca}$ & $0,7 \mathrm{Ba}$ & $0,7 \mathrm{Ba}$ \\
\hline Eucalyptus citriodora & $2,01 \mathrm{Ca}$ & $0,92 \mathrm{~Eb}$ & $0,8 \mathrm{Cb}$ & $0,7 \mathrm{Bb}$ & $0,7 \mathrm{Bb}$ \\
\hline $\mathrm{CV}(\%)$ & \multicolumn{5}{|c|}{10,67} \\
\hline dms & \multicolumn{5}{|c|}{0,24} \\
\hline
\end{tabular}

Média seguida de letra minúscula na linha e maiúscula na coluna não diferem entre si, pelo teste de tukey a 5\% de probabilidade. 
Tabela 3. Porcentagem de inibição de crescimento micelial (PICs) de Amphobotrys ricini em diferentes concentrações de fungicidas. Botucatu-SP.

\begin{tabular}{|c|c|c|c|c|c|c|c|c|c|c|c|c|}
\hline \multirow{3}{*}{ Tratamentos } & \multicolumn{12}{|c|}{ Concentração $(\mu \mathrm{L} / \mathrm{L})$} \\
\hline & \multicolumn{3}{|c|}{1} & \multicolumn{2}{|c|}{10} & & \multicolumn{3}{|c|}{100} & \multicolumn{3}{|c|}{1000} \\
\hline & \multicolumn{12}{|c|}{ ( $\mathrm{PIC} \%)$} \\
\hline Azoxystrobina & 43,20 & $\mathrm{~B}$ & $\mathrm{~b}$ & 42,50 & $\mathrm{~B}$ & $\mathrm{~b}$ & 53,40 & $\mathrm{~B}$ & $\mathrm{c}$ & 75,40 & $\mathrm{~A}$ & $\mathrm{~b}$ \\
\hline Clorotalonil & 5,00 & $\mathrm{~B}$ & $\mathrm{c}$ & 20,40 & $\mathrm{~B}$ & $\mathrm{c}$ & 100,00 & $\mathrm{~A}$ & $\mathrm{a}$ & 100,00 & A & $\mathrm{a}$ \\
\hline Tiofanato metílico & 100,00 & A & a & 100,00 & A & $\mathrm{a}$ & 100,00 & $\mathrm{~A}$ & $\mathrm{a}$ & 100,00 & A & $\mathrm{a}$ \\
\hline Carbendazim & 100,00 & $\mathrm{~A}$ & $\mathrm{a}$ & 100,00 & A & $\mathrm{a}$ & 100,00 & $\mathrm{~A}$ & $\mathrm{a}$ & 100,00 & $\mathrm{~A}$ & $\mathrm{a}$ \\
\hline Tebuconazole & 100,00 & $\mathrm{~A}$ & $\mathrm{a}$ & 100,00 & A & $\mathrm{a}$ & 100,00 & $\mathrm{~A}$ & $\mathrm{a}$ & 100,00 & $\mathrm{~A}$ & $\mathrm{a}$ \\
\hline Mancozeb & 24,00 & $\mathrm{C}$ & $\mathrm{bc}$ & 9,20 & $\mathrm{C}$ & $\mathrm{c}$ & 79,40 & $\mathrm{~B}$ & $\mathrm{ab}$ & 100,00 & A & $\mathrm{a}$ \\
\hline Iprodione & 100,00 & $\mathrm{~A}$ & $\mathrm{a}$ & 100,00 & A & $\mathrm{a}$ & 100,00 & $\mathrm{~A}$ & $\mathrm{a}$ & 100,00 & A & $\mathrm{a}$ \\
\hline Procimidone & 89,50 & A & $\mathrm{a}$ & 87,00 & A & $\mathrm{a}$ & 100,00 & A & $\mathrm{a}$ & 100,00 & A & $\mathrm{a}$ \\
\hline Carboxin + Thiram & 14,50 & $\mathrm{C}$ & $\mathrm{c}$ & 15,20 & $\mathrm{C}$ & $\mathrm{c}$ & 60,20 & $\mathrm{~B}$ & $\mathrm{bc}$ & 99,60 & $\mathrm{~A}$ & a \\
\hline $\mathrm{CV}(\%)$ & \multicolumn{12}{|c|}{13,44} \\
\hline $\mathrm{dms}$ & \multicolumn{12}{|c|}{10,49} \\
\hline
\end{tabular}

Médias seguidas de mesma letra maiúscula na linha e minúscula na coluna não diferem entre si pelo teste de Tukey a 5\% de probabilidade.

Tabela 4. Dose efetiva mediana $\left(\mathrm{ED}_{50}\right)$ de vários fungicidas relativos à inibição do crescimento micelial do isolado Amphobotrys ricini (L3). Botucatu-SP.

\begin{tabular}{cc}
\hline Tratamentos & $\begin{array}{c}\text { ED50 dos fungicidas } \\
(\boldsymbol{\mu L} / \mathbf{L})\end{array}$ \\
\hline Azoxystrobina & $>50$ \\
Clorotalonil & $10-50$ \\
Tiofanato metílico $*$ & $<1$ \\
Carbendazim * & $<1$ \\
Tebuconazole * & $<1$ \\
Mancozeb & $>50$ \\
Iprodione * & $<1$ \\
Procimidone & $<1$ \\
Carboxin +thiram & $>50$ \\
\hline
\end{tabular}

*tratamentos onde não foi possível elaborar a equação linear da reta ( $\mathrm{y}=100)$, devido ao fato de que em todas as concentrações testadas houve $100 \%$ de inibição do crescimento micelial, sendo apenas estimado o valor do ED50.

micelial do isolado de $A$. ricini nas concentrações estudadas com o i.a. tiofanato metílico.

Para o fungicida azoxystrobin, do grupo das estrobilurinas, Caldari Júnior (4) encontrou grande variação no crescimento micelial de isolados provavelmente ainda não expostos a esse fungicida, no qual o $\mathrm{ED}_{50}$ situou-se abaixo de $0,1 \mu \mathrm{L} / \mathrm{L}$ e para outros em $1000 \mu \mathrm{L} / \mathrm{L}$, indicando que esse composto não tem nenhuma ou pouca influência no crescimento micelial in vitro, discordando também do conceito de resistência de fungos a fungicidas; Sendo o mesmo comportamento observado neste trabalho, onde o $\mathrm{ED}_{50}$ foi maior que $50 \mu \mathrm{L} / \mathrm{L}$. Vários trabalhos relatam a eficiência de fungicidas benzimadizóis (26) e dicarboximidas (14) contra Botrytis cinerea, o que foi confirmado também neste trabalho.

Efeito de diferentes tratamentos na severidade de Amphobotrys ricini versus mamoneira, em ensaio conduzido no campo.

Na primeira semana, o melhor tratamento foi a pulverização de iprodione com severidade de $4,31 \%$, que não deferiu estatisticamente da testemunha não inoculada $(0,00 \%)$, seguido do tratamento procimidone
(21,88\%), a sequência continuou com o óleo essêncial de Cinnamomum zeylanicum (67,91\%), suspensão de esporos de Clonostachys rosea a $1 \times 10^{6}$ conídios/mL (71,56\%), suspensão de Trichoderma sp $(72,16$ \%), óleo de Cympobogon martini (82,94\%), testemunha inoculada $(89,91 \%)$ (Tabela 5 e Figura 1).

$\mathrm{Na}$ segunda semana a ordem da eficiência dos tratamentos quase não sofreu alteração, permanecendo o melhor tratamento a aplicação de iprodione (12,81\%), que não diferiu estatisticamente da testemunha não inoculada, sendo seguida pelo tratamento de procimidone $(60,16 \%)$. A alteração ocorreu em relação ao tratamento de óleo essencial de $C$. zeylanicum que mostrou maior severidade $(91,09 \%)$ quando comparado com os tratamentos de $C$. rosea e de Trichoderma sp., cujas severidades foram, respectivamente, de $81,25 \%$ e de $92,34 \%$, mas não houve diferença signficativa entre esses tratamentos.

Não houve alteração muito grande da severidade da doença em função dos tratamentos na terceira semana. O tratamento cuja aplicação foi Ipridione permaneceu como sendo o melhor com valor de $30,16 \%$, não diferindo da testemunha não inoculada $(0,00 \%)$, seguida novamente pelo tratamento de procimidone $(62,81 \%)$. Em relação aos demais tratamentos, os mesmos não mostraram diferenças significativas entre si, sendo os valores dos mesmos para C. zeylanicum $(94,06 \%)$, C. martini com $99,68 \%$, C. rosea $88,22 \%$ e 93,91\% para Trichoderma sp..

O tratamento a base de iprodione apresentou diferença estatística quando comparado com os demais tratamentos. Os resultados obtidos com o tratamento C. rosea foram inferiores aos do melhor tratamento (iprodione), possivelmente pelo fato de tratar-se de um método biológico, e, também, devido a aplicação realizada uma vez por semana. Em testes para comparar a eficiência de $C$. rosea e do fungicida Captan na proteção das flores do morango - principal via de invasão do fruto pelo $B$. cinerea, o fungo $\left(10^{6}\right.$ esporos $\left./ \mathrm{mL}\right)$ suprimiu a incidência de $B$. cinerea em estames de $93 \%$ para $79 \%$ e em frutos de $76 \%$ para $48 \%$, uma performance igual ou melhor que outros antagonistas e o fungicida. $\mathrm{O}$ antagonista $C$. rosea foi mais eficaz que Captan contra $B$. cinerea em frutos de oito diferentes cultivares de morango. Os tratamentos foram aplicados aos vasos ao amanhecer e antes do anoitecer, baseados na conjectura que orvalho e escuro podem facilitar a sobrevivência e a atividade dos 
Tabela 5. Avaliação da severidade de mofo-cinzento em racemos de mamoneira durante 3 semanas, em função da aplicação de diferentes tratamentos no campo. Botucatu-SP.

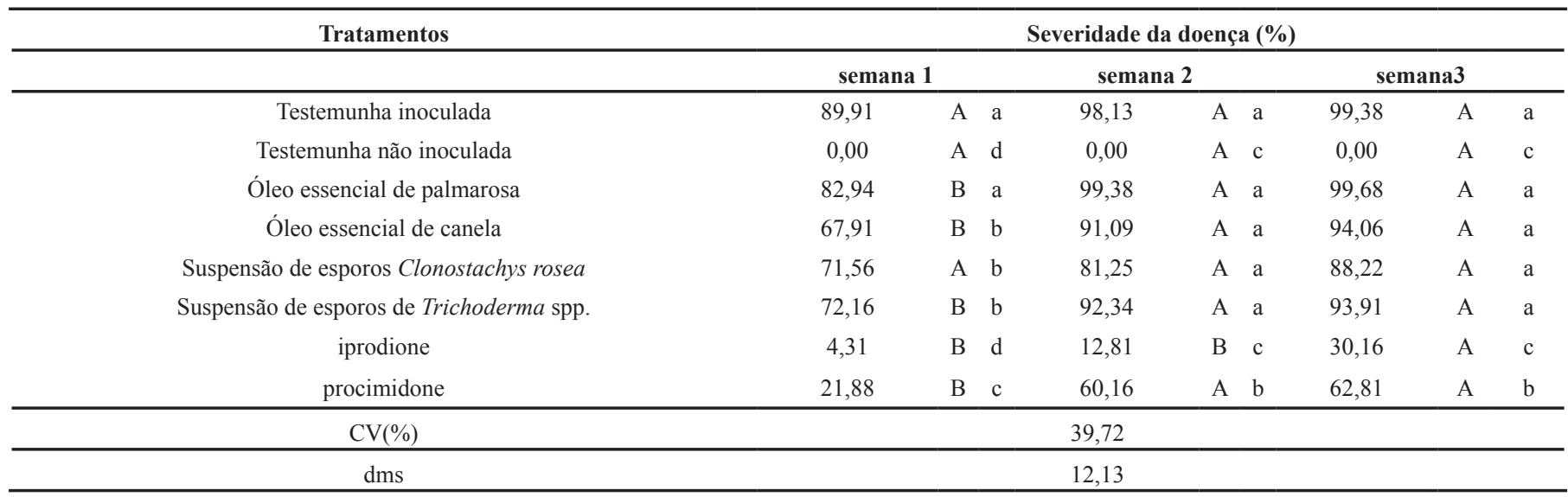

Médias seguidas de mesma letra minúscula na coluna e maiúscula na linha não diferem entre si pelo teste de Tukey a 5\% de probabilidade.
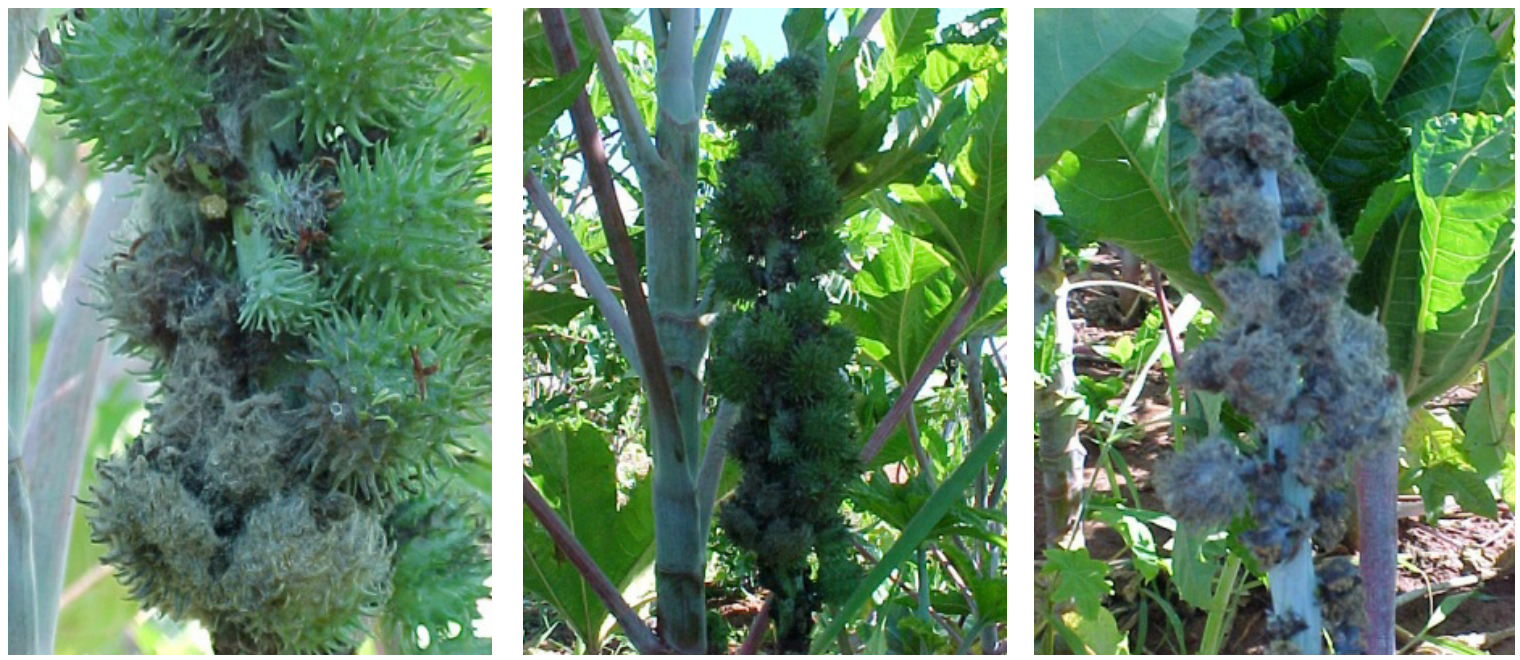

Figura 1. Sintomas de mofo cinzento causado pelo fungo Amphobotrys ricini em racemos de mamoneira.

organismos de biocontrole (28). Dessa forma, pelo menos duas aplicações semanais de C. rosea poderiam ser realizadas, ou até mesmo o uso associado às aplicações com o fungicida iprodione ou outro fungicida.

No trabalho desenvolvido por Lima \& Soares (13), 15 cultivares de mamoneira foram avaliados quanto à resistência ao mofo-cinzento. Uma das cultivares avaliadas foi a Guarani, que apresentou índice de doença na primeira e segunda avaliação de $40,83 \%$ e $48,38 \%$, respectivamente, sendo considerado com resistência moderada ao $A$. ricini. Na avaliação da intensidade da doença em cinco cultivares de mamoneira (Guarany, AL Guarany 2002, Mirante 10, hibrido Lyra e híbrido 2), sob diferentes arranjos populacionais, foi observado que todas as cultivares comportaram-se como suscetíveis, com variação de incidência de frutos infectados de $25 \%$ (AL Guarany) a $48 \%$ (Guarany). Resultados similares foram encontrados por Rego Filho et al. (20), onde a cultivar AL Guarany não diferiu estatisticamente do híbrido Lyra, apresentando incidência de mofo cinzento de $20,00 \%$ e $25,50 \%$, respectivamente.

Vários são os fatores envolvidos nos resultados. Do ponto de vista genético não podem ser esquecidos que genótipos cujas cápsulas possuem poucos acúleos são mais resistentes ao ataque do mofo cinzento, enquanto que aqueles com cápsulas com muitos acúleos comportaram-se como os mais suscetíveis ao patógeno, o que foi comprovado por Lima \& Soares (13). As dificuldades encontradas na obtenção de linhagens de mamoneira com resistência a $A$. ricini podem ser devidas à produção de enzimas hidrolíticas, possivelmente enzimas pécticas e celulolíticas, que decompõem os tecidos dos frutos infectados (29), bem como devido a maior quantidade de açúcares solúveis presente nas cápsulas de mamoneira suscetíveis (19). Portanto, em estudos seguintes visando à obtenção de cultivares de mamoneira com certo nível de resistência a $A$. ricini, faz-se necessário investigar com maior detalhamento as características morfológicas, os teores de açúcares solúveis da planta e a produção de enzimas hidrolíticas por este patógeno.

Dessa maneira, vários são os fatores que devem ser levados em consideração no que diz respeito ao controle do mofo-cinzento da mamoneira, um trabalho conjunto visando métodos de controle tanto químicos, quanto alternativos e a busca por materiais com níveis de resistência genética aceitáveis.

Quanto a inibição do crescimento micelial de $A$. ricini, os melhores tratamentos com os óleos essenciais foram os com $C$. martini e $C$. zeylanicum em todas as concentrações testadas, enquanto que com os fungicidas, em todas as concentrações testadas os melhores foram os ingredientes ativos tiofanato metílico, carbendazin, tebuconazole e iprodione.

No campo, o tratamento com o fungicida iprodione (Rovral) foi 
o melhor quanto ao controle da doença quando comparados com os tratamentos biológico e alternativo (óleos essenciais).

\section{REFÊRENCIAS BIBLIOGRAFICAS}

1.Abreu, C. L. M. Controle de Alternaria solani em tomateiro (Lycopersicon esculentum) com óleos essenciais. 2006. 71f. Tese (Doutorado em Agronomia / Horticultura)-Faculdade de Ciências Agronômicas, Universidade Estadual Paulista, Botucatu.

2. Bollen, J.; Fucks, A. On the specificity of the in vitro and in vivo antifungal activity of benomyl. Netherland Journal of Plant Pathology, v.76, p.299-313,1970.

3. Brent, K J. Fungicide resistance in crop pathogens: how can it be managed. Brussels: Global Crop Protection Federation, 1995. 48p.

4. Caldari Júnior, P. Caracterização morfológica, esporulação e sensibilidade a fungicidas de isolados de Botrytis cinerea de flores e plantas ornamentais. 1998. 51f. Dissertação (Mestrado em Agronomia / Fitopatologia)- Escola Superior de Agricultura Luiz de Queiroz, Universidade de São Paulo, Piracicaba.

5. Chagas, H. A.; Basseto, M. A.; Rosa, D. D.; Furtado, E. L.; Zanotto, M. D. Escala diagramática para avaliação de mofo cinzento (Amphobotrys ricini) da mamoneira (Ricinus communis L.). Summa Phytopathológica, Botucatu, v.36, n.2, p. 164-167. 2010.

6. Chierice, G.O.; Claro Neto, S. Aplicação Industrial do óleo. In: Azevedo, D.M.P. de; Lima, E.F. (Ed.). O agronegócio da mamona no Brasil. Brasília, DF: Embrapa Informação Tecnológica, 2001. p. 89-120.

7. Edington, L.V.; Khen, K.L.; Barron, G.L. Fungitoxic spectrum of benzimidazole compounds. Phytopathology, Saint Paul, v.61, p.4244, 1971.

8. Fisher, I. H. Seleção de plantas resistentes e de fungicidas para o controle da "morte prematura" do maracujazeiro, causada por Nectria haematococca e Phytophthora parasítica. 2003. 48f. Dissertação (Mestrado em Agronomia / Fitopatologia)- Escola Superior de Agricultura Luiz de Queiroz, Universidade de São Paulo, Piracicaba.

9. Fornazieri Junior, A. Mamona: uma rica fonte de óleo e de divisas. São Paulo: Ícone, 1986. 72 p.

10. Kataria, H.R.; Grover, R.K. Comparison of fungicides for the control of Rhizoctonia solani causing damping-off of mung bean (Phaseolus aureusi). Annual Applied Biology, Camberra, v. 88, p.257-263, 1978.

11. Kimura, M.K. Sensibilidade in vitro de Botrytis cinerea a fungicidas. 1999. 132f. Dissertação (Mestrado em Fitopatologia) - Universidade Federal de Lavras, Lavras.

12. Lima, E.F.; Araújo, A.E.; Batista, F.A.S. Doenças e seu controle. In.: Azevedo, D.M.P. de; Lima, E.F. (Ed.). O agronegócio da mamona no Brasil, Brasília, DF: Embrapa Informação Tecnológica, 2001. p. 191-212.

13. Lima, E.F. ; Soares, J.J. Resistência de cultivares da mamoneira ao mofo cinzento causado por Botrytis ricini. Fitopatologia Brasileira, Lavras, v. 15, n.1, p.96-97,1990.

14. Lorenz, G. Dicarboximide fungicides: history of resistence development and monitoring methods. In: DELP, C.J. Fungicide resistance in North
America 2. ed. Saint Paul: APS Press, 1994. cap.4, p. 45-51.

15. Mccalley, D.; Torres-Grifol, J. F. Analysis of volatiles in good and bad conditions by gas chromatography and gas chromatography-mass spectrometry. Analyst, Washington, DC: v. 117, p. 721-725, 1992.

16. Menten, J.O.M.; Minussi, C.C.; Castro, C.; Kimati, H. Efeito de alguns fungicidas no crescimento micelial de Macrophomina phaseolina (Tass.) Goid. "in vitro". Fitopatologia Brasileira, Brasilia D.F., v. 1, n. 2, p. 57-66, 1976.

17. Montes-Belmont, R.; Carvajal, M. Control of Aspergillus flavus in maize with plant essential oils and their components. Journal of Food Protection, Annes, v. 61, p. 616-619, 1998.

18. Norman, S.; Craft, C. C.; Davis, P. L. Volatiles from injured and uninjured Valencia oranges at different temperatures. Journal Food Science, Chicago, v. 32 , p. $656-659,1967$

19. Orellana, R.G.; Thomas, C.A. Nature of predisposition of castorbeans to Botrytis.I. Relation of leachable sugar and certain other biochemichal constituents of the capsule to varietal susceptility. Phytopathology, Saint Paul, v. 52, p. 533-538, 1962.

20. Rêgo Filho, L. M.; Bezerra Neto, F. V.; Santos, Z. M. Avaliação da incidência de mofo cinzento em genótipos de mamoneira no período outono-inverno em Campos dos Goytacazes - RJ. In: Congresso Brasileiro de Plantas Oleaginosas, Óleos, Gorduras e Biodiesel. 4, 2007, Varginha-MG. Anais. Lavras: Universidade Federal de Lavras, 2007. 1 CD-ROM.

21. Salgado, A.P.S.P.; Cardoso, M.G.; Souza, P.E.; Souza, J.A.; Abreu, C.M.P.; Pinto, J.E.B.P. Avaliação da atividade fungitóxica de óleos essenciais de folhas de Eucalyptus sobre Fusarium oxysporum, Botrytis cinerea e Bipolaris sorokiniana. Ciência Agrotécnica, Lavras, v. 27, n.2, p. 249-254, 2003.

22. Savy Filho, A. Melhoramento da mamona. In: BORÉM, A. Melhoramento de espécies cultivadas. Viçosa: Editora UFV, 1999. p. 383-407.

23. Schwan-Estrada, K. R. F.; Stangarlin, J. R.; Cruz, M. E. da S. Uso de extratos vegetais no controle de fungos fitopatogênicos. Revista Floresta, Curitiba, v. 30, n. $1 / 2$, p. $129-137,2000$

24. Singh, R. S.; Pathak, M. G.; Bodoli, D. W. Response of Java citronella cultivars to nitrogen under Jorhat conditions. Indian Perfurmer, Kampur, v. 24 , n. 4 , p. $192-198,1980$.

25. Singh, H. B. et al. Cinnamon bark oil, a potent fungitoxicant against fungi causing respiratory tract mycoses. Allergy, Copenhagen, v.50 p. 995-999, 1995.

26. Smith C.M. Benzamidazole fungicides. In: DELP, C.J. (Ed.) Fungicide Resistencia in North America. 2. ed. St. Paul: APS Press, 1994. cap.3, p. 23-44.

27. Souza, S. M. C. et al. Avaliação de óleos essenciais de condimentos sobre o desenvolvimento micelial de fungos associados a produtos de panificação. Ciências Agrotécnicas, Lavras, v. 28, n. 3, p. 685-690, maio/jun., 2004.

28. Sutton, J.C.; Li, De-Wei; Peng, G.; Yu, H.; Zhang, P.; Valdebenito Sanhueza, R.M. Gliocladium roseum: a versatile adversary of Botrytis cinerea in crops. Plant Disease, Saint Paul, v. 81, p. 316-328, 1997.

29. Thomas, C.A.; Orellana, R.G. Biochemical tests indicative of reaction of castor bean to Botrytis. Science, Washington, DC: v. 139, p. 334-335, 1963.

30. Weindling, R. Trichoderma lignorum as a parasite of other soil fungi. Phytopathology, Saint Paul, v. 22, p. 837-845. 1932. 